\title{
Modeling Chemical Kinetics in Solid State Reactions
}

\author{
J.A. Huidobro, I. Iglesias, B.F. Alfonso, C. Trobajo, and J.R. Garcia
}

\begin{abstract}
This work deals with the kinetics of thermally stimulated processes which take place in the solid state phases. The activation energy of the solid is calculated using several methods of different families of isoconversional methods (differential, integral and incremental). A model of the kinetics is obtained by a method independent from the procedure used to compute the activation energy and it is analysed in three theoretical simulations as well as the thermal degradation of $\mathrm{FeNH}_{4}\left(\mathrm{HPO}_{4}\right)_{2}$. The reconstructed $\alpha-T$ curves of the simulations and the experimental case indicates that the model works properly.
\end{abstract}

\section{Modeling Kinetics}

The study of kinetics in solid state reactions explains the mechanism of the chemical processes as well as the meaning of the related parameters. It provides qualitative and quantitative information on phase transformations, crystallization, thermal decomposition, etc. Several analysis techniques such as Thermogravimetric Analysis (TGA), have been developed to measure physical and chemical changes.

A simple stimulated thermal reaction follows a scheme in the form $\operatorname{Re} \rightarrow P+S$ where $R e$ is the reactant, $P$ the solid product and $S$ is the solvent or water vapour. The reaction progress is given by the extent of conversion $\alpha(t)=\left(m_{0}-m(t)\right) /\left(m_{0}-m_{f}\right)$

\footnotetext{
J.A. Huidobro $(\square)$

Departamento de Matematicas, Universidad de Oviedo, Gijón, Spain

e-mail: jahuidobro@uniovi.es

I. Iglesias • B.F. Alfonso

Departamento de Fisica, Universidad de Oviedo, Gijón, Spain

e-mail: iis@uniovi.es; mbafernandez@uniovi.es

C. Trobajo • J.R. Garcia

Departamento de Quimica Organica e Inorganica, Universidad de Oviedo, Oviedo, Spain

e-mail: ctf@uniovi.es; jrgm@uniovi.es
} 
where $m(t)$ is the mass of Re at time $t$ and $m_{0}$ and $m_{f}$ are the initial and final masses, respectively. These reactions are commonly described by the equation

$$
\frac{d \alpha}{d t}=A \exp \left(-\frac{E}{R T}\right) f(\alpha)
$$

where $T$ is the temperature, $R$ the universal gas constant, $A$ the pre-exponential factor, $E$ the activation energy and $f(\alpha)$ the model function [5].

The knowledge of $A, E$ and $f(\alpha)$, the called kinetic triplet, allows solving Eq. (1) and so a description of the process can be obtained. In the model-fitting methods, the obtention of the kinetic triplet is based on the determination of the model function by fitting several reaction models to the experimental data and then the coefficients $A$ and $E$ are computed. But different forms of $f(\alpha)$ with disparate values of $A$ and $E$ can be fitted to the data and then these methods are not recommended [6].

In isoconversional methods the activation energy is computed without knowing the model function or the pre-exponential factor [7]. Consequently, a model of the process based on Eq. (1) cannot be obtained. Some authors [4, 8] have proposed different methods in order to calculate the product $A f(\alpha)$, considered as a sole factor, depending on how the activations energy has been computed. The main purpose of this study is to analyse the behaviour of a method to compute $A f(\alpha)$, independent of the procedure used to compute the activation energy, when it is applied to three theoretical simulations as well as the thermal degradation of $\mathrm{FeNH}_{4}\left(\mathrm{HPO}_{4}\right)_{2}$.

Mechanisms of chemical transformations are indeed complicated, they usually involve more than a single reaction. Then, Eq. (1) must be understood as an approximation to describe the process, the kinetic parameters are considered as apparent parameters and their physical meaning should be carefully analysed.

One of the simplest isoconversional methods is that proposed by Friedman (FR) [2], which is a differential isoconversional method. For a constant heating rate program of temperature $T=T_{0}+\beta t$ and taking logarithms, Eq. (1) turns into

$$
\ln \left(\frac{d \alpha(T)}{d T} \beta\right)=\ln (A f(\alpha(T)))-\frac{E}{R T}
$$

where now $\alpha(T)$ represents the dependence of the extent of conversion respect to the temperature. Several runs with different heating rates $\beta_{i}, i=1, \ldots, n$ with $n \geq 3$ are carried out and $n$ experimental $\alpha-T$ curves are obtained. Thus, for a fixed value of $\alpha$ and from each experimental curve, values for $T_{i}$ and $d \alpha\left(T_{i}\right) / d T$ are obtained. Then, from Eq. (2), the points $\left.\left(1 / T_{i}, \ln \left(d \alpha\left(T_{i}\right) / d T\right) \beta_{i}\right)\right)$ belong to a straight line whose slope is $-E_{\alpha} / R$. The activation energy $E_{\alpha}$ can be obtained by fitting to the experimental data.

Wu et al. [8] extended this method by computing not only $E$ but also $\ln (A f(\alpha(T)))$ and then, the product $A f(\alpha)$ is known and the differential Eq. (1) can be solved.

Generally, one drawback of this method is its sensitivity to noise that can come from numerical differentiation or experimental measures. A method (MFR) to 
diminish this effect was proposed in [3] where Eq. (2) is considered for more values of $\alpha$. Then, by fitting to the experimental data, the activation energy is computed.

Less sensitive to noise are integral isoconversional methods that consider an integral form of Eq. (1) but $\exp (-E /(R T))$ does not have a suitable antiderivative and some approximations have been proposed. They are based on assuming $E$ is constant over the whole process and this is not very common. One of these is the generalized Kissinger method [1] (KAS), widely used. Vyazovkin [5] introduced an non-linear method (Vyaz) by integration of Eq. (1) over $\left[\alpha^{*}-\Delta \alpha^{*}, \alpha^{*}\right]$ and Samuelsson [4] computed $A f(\alpha)$, assuming it is constant over the interval.

A different idea, where this assumption is not necessary, is to compute the factor $A f(\alpha)$ directly from Eq. (1). Assuming the activation energy is known, for a fixed value of $\alpha$, the product $A f(\alpha)$ can be obtained by fitting to the experimental data. Then, the differential Eq. (1) can be solved and a model is obtained. In this work, the four aforementioned methods were used to determine the activation energy and four set of values $E_{\alpha}-\alpha$ were obtained. For each method, the corresponding values of $A f(\alpha)$ were computed and model of the kinetics was achieved.

\section{Results and Conclusions}

This procedure to obtain a kinetic model has been implemented in Matlab and it was applied to three theoretical simulations. In all cases four constant heating rates were applied to generate the simulated data. In the first case, a one-step model with a first-order model function $f(\alpha)=1-\alpha$ and Arrhenius parameters $A=10^{9} \mathrm{~min}^{-1}$ and $E=10^{2} \mathrm{~kJ} \mathrm{~mol}^{-1}$ were considered. The four methods used to compute the activation energy provide similar values. Using them, the product $A f(\alpha)$ was computed and then $\alpha-T$ curves were plotted by solving the general kinetic differential equation.

In the second simulation, a parallel two-step, equally weighted, case was analysed being $f_{1}(\alpha)=1-\alpha, A_{1}=10^{12} \mathrm{~min}^{-1}$ and $E_{1}=167 \mathrm{~kJ} \mathrm{~mol}^{-1}$ and $f_{2}(\alpha)=1-\alpha, A_{2}=10^{26} \mathrm{~min}^{-1}$ and $E_{2}=352 \mathrm{~kJ} \mathrm{~mol}^{-1}$. The third simulation analysed an Avrami-Erofeev model function $f(\alpha)=4(1-\alpha)\left[-\ln (1-\alpha)^{3 / 4}\right]$ with $A=10^{2} \mathrm{~min}^{-1}$ and $E=20.9 \mathrm{~kJ} \mathrm{~mol}^{-1}$. Figure 1 shows the simulated and the reconstructed $\alpha-T$ curves for the three simulations. In all cases a good agreement was achieved.

Finally, thermogravimetric analysis of the thermal degradation of $\mathrm{FeNH}_{4}\left(\mathrm{HPO}_{4}\right)_{2}$ was conducted in a $\mathrm{N}_{2}$ dynamic atmosphere, using a Mettler-Toledo TGA/SDTA851 ${ }^{e}$, at four different heating rates. As can be seen in Fig. $2 \mathrm{a}$, the process occurs in two consecutive stages, the on-line mass spectrometric analysis indicates that the material firstly loses water about $600 \mathrm{~K}$ and secondly, at about $900 \mathrm{~K}$, water and ammonia. The $E-\alpha$ plot, displayed in Fig. 2b, shows this behaviour. The experimental data are satisfactorily reconstructed (Fig. 2c)

In conclusion, the product $A f(\alpha)$ has been computed by a method independent of how the activation energy has been obtained. Then, the general kinetic differential 


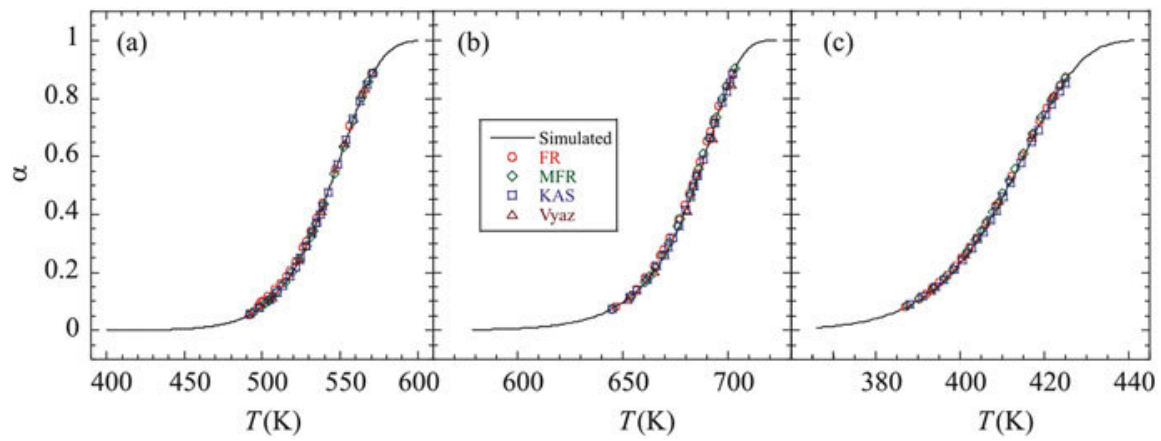

Fig. 1 Comparison of the simulated and reconstructed $\alpha-T$ curves for $\beta=8 \mathrm{~K} \mathrm{~min}^{-1}$. Simulations 1, 2 and 3 in (a), (b) and (c), respectively
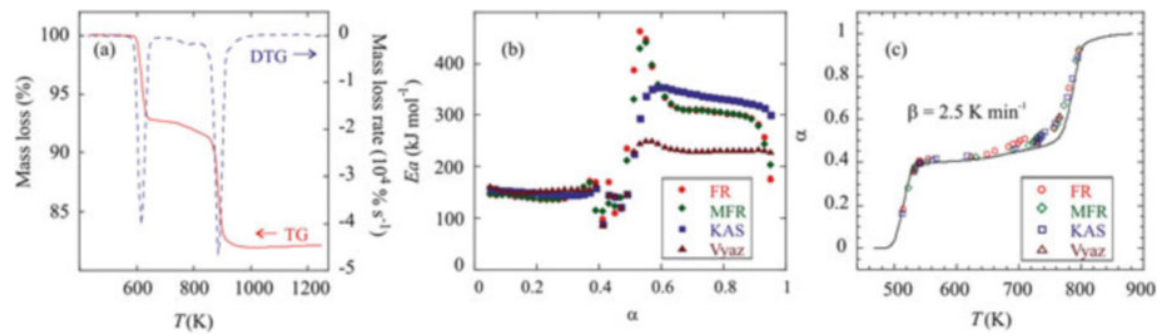

Fig. 2 TG and DTG curves of $\mathrm{FeNH}_{4}\left(\mathrm{HPO}_{4}\right)_{2}$ obtained at $10 \mathrm{~K} \mathrm{~min}^{-1}$ heating rate (a); activation energy versus extent of conversion (b); experimental (line) and reconstructed (points) $\alpha-T$ curves for $\beta=2.5 \mathrm{~K} \mathrm{~min}^{-1}$ (c)

equation can be solved overcoming the ambiguity of the model-fitting methods. In this way, a discrete model that can be used to describe kinetics in solid state processes. This model has worked efficiently in the description of the theoretical simulations studied and in the thermal decomposition of $\mathrm{FeNH}_{4}\left(\mathrm{HPO}_{4}\right)_{2}$.

Acknowledgements This work was supported by Ministerio de Economia y Competitividad (MAT2013-40950-R, MAT2011-27573-C04-02), Gobierno del Principado de Asturias (GRUPIN14-060 and GRUPIN14-037), and FEDER.

\section{References}

1. Akahira, T., Sunose, T.: Joint Convention of four electrical institutes. Research report (Chiba Institute of Technology) Sci. Technol. 16, 22-31 (1971)

2. Friedman, H.: Kinetics of thermal degradation of charge-forming plastics from thermogravimetry. Application to a phenolic plastic. J. Polym. Sci. Part C 6, 183-195 (1964)

3. Huidobro, J.A., Iglesias, I., Alfonso, B.F., Trobajo, C., Garcia, J.R.: Reducing the effects of noise in the calculation of activation energy by the Friedman method. Chemom. Intell. Lab. Syst. (Chiba Institute of Technology) Sci. Technol. 16, 22-31 (1971) 
4. Samuelsson, L.N., Moriana, R., Babler, M.U., Ek, M., Engvall, K.: Model-free rate expression for thermal decomposition processes: The case of microcrystalline cellulose pyrolysis. Fuel 143, 438-447 (2015)

5. Vyazovkin, S., Dollimore, D.: Linear and nonlinear procedures in isoconversional computations of the activation energy of nonisothermal reactions in solids. J. Chem. Inf. Comput. Sci. 151, $146-152$ (1996)

6. Vyazovkin, S., Wight, C.A.: Kinetics in solids. Annu. Rev. Phys. Chem. 48, 125-149 (1997)

7. Vyazovkin, S., Burnham, A.K., Criado, J.M., Pérez-Maqueda, L.A., Popescu, C., Sbirrazzuoli, N.: ICTAC Kinetic Committee recommendations for performing kinetic computations on thermal analysis data. Thermochim. Acta 520, 1-19 (2011)

8. Wu, W., Cai, J., Liu, R.: Isoconversional kinetic analysis of distributed activation energy. Model processes for pyrolysis of solid fuels. Ind. Eng. Chem. Res. 52, 14376-14383 (2013) 